\title{
A nationwide survey to measure practice variation of catheterisation management in patients undergoing vaginal prolapse surgery
}

\author{
R. A. Hakvoort • M. P. Burger • M. H. Emanuel • \\ J. P. Roovers
}

Received: 14 October 2008 / Accepted: 17 February 2009/Published online: 10 March 2009

(C) The Author(s) 2009. This article is published with open access at Springerlink.com

\begin{abstract}
Introduction and hypothesis Urinary catheterisation following vaginal prolapse surgery causes inconvenience for patients, risk of urinary tract infections and potentially longer hospitalisation. Possibly, practice variation exists concerning diagnosis and management of abnormal postvoid residual (PVR) volume implying suboptimal treatment for certain subgroups.

Methods Nationwide questionnaire-based survey.

Results Post-operatively, 77\% performed transurethral indwelling catheterisation, $12 \%$ suprapubic catheterisation and $11 \%$ intermittent catheterisation. Catheterisation was applied 3 days (1-7 days) following anterior repair and 1 day (1-3 days) following all other procedures. The median cut-off point for abnormal PVR was $150 \mathrm{~mL}$ (range 50-250 mL). Treatment of abnormal PVR consisted mostly of prolonging transurethral indwelling catheterisation for 2 days (range $1-5$ days; $57 \%$ ), 29\% by intermittent and $12 \%$ by suprapubic catheterisation. Antibiotics were administered by $21 \%$ either routinely or based on symptoms only.

Conclusions Due to insufficient evidence and suboptimal implementation of available evidence, practice variation in catheterisation regimens is high.
\end{abstract}

R. A. Hakvoort $(\bowtie) \cdot$ M. H. Emanuel

Department of Obstetrics and Gynaecology, Spaarne Hospital, P.O. Box 770, 2130 AT Hoofddorp, The Netherlands e-mail: rhakvoort@spaarneziekenhuis.nl

M. P. Burger $\cdot$ J. P. Roovers

Department of Obstetrics and Gynaecology,

Academic Medical Center, University of Amsterdam,

Amsterdam, The Netherlands
Keywords Pelvic floor repair · Pelvic organ prolapse . Survey $\cdot$ Urinary catheterisation

\section{Introduction}

Urinary catheterisation is a frequently performed procedure in hospitalised patients [1]. Its use is accompanied by an increased risk of urinary tract infections, inconvenience for patients, costs and often prolonged hospitalisation [1, 2]. Therefore, the judicious use of catheterisation is important. In practice, the initiation is often poorly indicated and continuation of catheterisation is often longer than intended [3]. Reported reasons for this are negligence (i.e. forgetting the presence of a catheter) and convenience for nursing staff $[1,3]$. The importance of documentation of catheterisation regimens has been emphasised in order to diminish duration of catheterisation [4].

Most gynaecologists routinely catheterise their patients following pelvic organ prolapse (POP) surgery. In this patient category, catheterisation is also frequently prolonged for several days post-operatively. Hilton and colleagues evaluated catheterisation strategies among gynaecologists in the UK and reported that initial catheterisation time varied from 1 to 7 days [5]. The use of these prolonged catheterisation protocols may be prompted by the presence of a vaginal gauze post-operatively but can also be thought to be mandatory as post-operative pain, anaesthesia-related procedures and paraurethral oedema or innervation trauma can prevent optimal emptying of the bladder. Limited evidence or inadequate implementation of available evidence may result in practice variation regarding catheter regimens. Such practice variation is an unwanted feature as it might signify that not all patients receive optimal treatment. 
We present the data of a nationwide survey assessing the presence and content of catheterisation protocols in patients undergoing vaginal POP surgery. We also evaluated how gynaecologists defined and managed abnormal postvoid residual (PVR) volumes and urinary tract infections, as the last is the most common complication of catheterisation.

\section{Materials and methods}

A survey was performed among all 99 general and academic hospitals in The Netherlands. A mailed questionnaire was sent to the gynaecologist responsible for urogynaecology in each hospital. A reminder was sent to non-responders 3 months after the first request.

The questionnaire addressed the following five topics:

Topic 1: Characteristics of the hospital: (1) university or non-university hospital, (2) teaching or nonteaching hospital, (3) employment of a gynaecologist specialised in urogynecology, (4) existence of a bladder catheterisation protocol or not.

Topic 2: Pre-operative and post-operative catheterisation management: (1) routinely emptying of the bladder before surgery or not, (2) routine administration of antibiotics before surgery or not, (3) routinely placing of a vaginal gauze post-operatively or not.

Topic 3: Protocolised catheter regimens following different types of vaginal POP surgery.

Topic 4: Abnormal postvoiding residual volume: (1) the definition of abnormal PVR (cut-off point of acceptable residual bladder volume and whether this residual volume had to exceed the cut-off point at one or two different occasions), (2) the technique to measure PVR (bladder scan, catheterisation or ultrasound), and (3) management of abnormal PVR.

Topic 5: The definition of urinary tract infection and the indications for the administration of antibiotics.
Statistics

Descriptive data analysis was performed using SPSS 15.0. (SPSS Statistics UK, SPSS Inc, Chicago, IL, USA).

\section{Results}

A total of 99 questionnaires were distributed. After two requests, 92 of the 99 hospitals had responded, which represents an overall response rate of $93 \%$.

Table 1 shows the characteristics of the responding hospitals. In the majority (90\%) of the hospitals, a gynaecologist was employed with a special interest in urogynaecology. Only five hospitals did not have a catheterisation protocol, of which one was a teaching hospital.

Table 2 shows the responses to questions related to preoperative and post-operative management. Before surgery, $69 \%$ of the gynaecologists routinely empty the bladder using transurethral catheterisation. Of the respondents, $83 \%$ routinely administer antibiotics pre-operatively, $13 \%$ do not and the remaining $4 \%$ administer antibiotics per-operatively only when the abdominal cavity has been opened. Seventyfive percent of gynaecologists routinely insert vaginal gauze post-operatively. No respondent removed the gauze later than the morning of the next post-operative day. Most gynaecologists $(77 \%)$ use a transurethral indwelling catheter for post-operative bladder drainage, $12 \%$ perform suprapubic catheterisation and $11 \%$ immediately starts intermittent catheterisation.

Table 3 shows median durations of transurethral indwelling catheterisation following different types of vaginal POP surgery. The median duration of initial catheterisation following anterior repair was 3 days (range 1-7 days). For the combination of anterior and posterior repair, the range was $1-5$ days with a median of 3 days. Following posterior repair, this was 1 day (range 0-3 days).

Table 4 shows the responses to questions addressing abnormal PVR and its management. The cut-off value for an abnormal PVR varied from 50 to $250 \mathrm{~mL}$ with a median volume of $150 \mathrm{~mL}$. Mostly (83\%), this residual volume was

Table 1 Characteristics of respondents $(n=92)$

\begin{tabular}{|c|c|c|c|c|c|c|}
\hline Type of department & Number response & Percent response & Urogynaecologist present & Percentage & Protocol present & Percentage \\
\hline University & 8 & 100 & 8 & 100 & 8 & 100 \\
\hline Teaching & 33 & 92 & 30 & 90 & 32 & 97 \\
\hline Non-teaching & 51 & 93 & 45 & 88 & 47 & 85 \\
\hline Total & 92 & 93 & 83 & 90 & 87 & 95 \\
\hline
\end{tabular}

Total number of university hospitals, teaching hospitals and non-teaching hospitals are, respectively 8,36 and 55 
Table 2 Standard peri-operative protocol

\begin{tabular}{|c|c|c|}
\hline Protocol & $n$ & Percent \\
\hline \multicolumn{3}{|l|}{ Routinely emptying bladder pre-operatively $(n=92)$} \\
\hline Always & 63 & 69 \\
\hline \multicolumn{3}{|l|}{ Type of catheter } \\
\hline Indwelling catheter & 17 & \\
\hline Intermittent catheter & 46 & \\
\hline Never & 29 & 31 \\
\hline \multicolumn{3}{|l|}{ Standard type of post-operative catheterisation $(n=92)$} \\
\hline Transurethral & 71 & 77 \\
\hline Suprapubic & 11 & 12 \\
\hline Intermittent & 10 & 11 \\
\hline \multicolumn{3}{|l|}{ Insertion of a vaginal gauze post-operatively $(n=76)$} \\
\hline Standard insertion & 57 & 75 \\
\hline \multicolumn{3}{|l|}{ When indicated } \\
\hline Higher amounts of blood loss per-operatively & 6 & 8 \\
\hline After surgery on more than one compartment & 12 & 16 \\
\hline Never & 1 & 1 \\
\hline \multicolumn{3}{|c|}{ Routine administration of antibiotics pre-operatively $(n=92)$} \\
\hline Yes & 76 & 83 \\
\hline No & 12 & 13 \\
\hline No, only when peritoneal cavity has been opened & 4 & 4 \\
\hline
\end{tabular}

measured using a bladder scanning device. In $60 \%$ of the hospitals, the residual volume was measured on two different occasions after removal of the catheter before an abnormal PVR was considered. When an abnormal PVR occurred after removal of the initial transurethral catheter, 57\% inserted a transurethral catheter for the second time for 2 to 3 days, whereas $29 \%$ of the respondents started intermittent catheterisation and $12 \%$ chose suprapubic catheterisation. Two percent did not respond to this question.

Table 5 shows the criteria for urinary tract infection and the indications for administration of antibiotics. Twenty-one percent of respondents administered antibiotic treatment without urinary analysis or culture. Of the respondents, $66 \%$ depended on urine analysis (dipstick testing) as the minimal requirement to start antibiotic treatment. Seventyfour percent of this group found the presence of nitrite only sufficient to consider an infection present and to initiate antibiotic treatment. Only 13\% requested a positive urinary culture before treatment was started. In case urine culture was demanded for, more than $70 \%$ used a cut-off point of $>10^{5}$ colony-forming units to consider the urine culture to be positive.

\section{Discussion}

A nationwide survey was performed to assess the presence and content of catheterisation protocols after vaginal prolapse surgery and to evaluate how gynaecologists define and manage abnormal PVR as well as urinary tract infections.

The proportion of hospitals with a catheterisation protocol was high (95\%); however, the content of these protocols varied to a great extent. The duration of initial standard catheterisation, cut-off levels for the definition of abnormal PVR and diagnostic and therapeutic criteria for urinary tract infections varied widely.

Table 3 Post-operative standard durations of transurethral indwelling catheterisation

\begin{tabular}{lrrrrr}
\hline $\begin{array}{l}\text { Duration of standard } \\
\text { initial catheterisation }\end{array}$ & \multicolumn{6}{c}{ Different surgical procedures } \\
\cline { 2 - 6 } & AR & PR & APR & $\begin{array}{l}\text { SSF or other uterus } \\
\text { suspension techniques }\end{array}$ & VH \\
& & & & 1 & 6 \\
0 & 0 & 7 & 0 & 43 & 73 \\
1 & 5 & 68 & 5 & 23 & 9 \\
2 & 31 & 13 & 32 & 15 & 3 \\
3 & 46 & 4 & 45 & 1 & 0 \\
4 & 3 & 0 & 3 & 0 & 0 \\
5 & 6 & 0 & 5 & 0 & 0 \\
6 & 0 & 0 & 0 & 1 & 0 \\
7 & 1 & 0 & 0 &
\end{tabular}

Values are numbers of hospitals

$A R$ anterior repair (with or without mesh), $P R$ posterior repair (with or without mesh), $A P R$ anterior and posterior repair (with or without mesh), $S S F$ sacrospinous ligament fixation, $V H$ vaginal hysterectomy 
Table 4 Protocol after removal of initial catheter $(n=92)$

\begin{tabular}{|c|c|c|}
\hline Protocol & $n$ & Percent \\
\hline \multicolumn{3}{|l|}{ Technique of measurement residual volume } \\
\hline Ultrasound & 2 & 2 \\
\hline Bladder scan & 76 & 83 \\
\hline Catheter & 14 & 15 \\
\hline \multicolumn{3}{|l|}{ Cut-off point for residual volume $(\mathrm{mL})$} \\
\hline 50 & 1 & 1 \\
\hline 100 & 28 & 30 \\
\hline 150 & 38 & 41 \\
\hline 200 & 18 & 20 \\
\hline 250 & 7 & 8 \\
\hline \multicolumn{3}{|c|}{ The number of measurements required before urinary retention is diagnosed } \\
\hline One measurement directly after first voiding & 37 & 40 \\
\hline Two measurements after two separate voidings & 55 & 60 \\
\hline \multicolumn{3}{|l|}{ Type of catheter after diagnosis retention } \\
\hline Indwelling transurethral & 52 & 57 \\
\hline 1 day & 9 & \\
\hline 2 days & 23 & \\
\hline 3 days & 19 & \\
\hline 4 days & 0 & \\
\hline 5 days & 1 & \\
\hline Sterile intermittent catheterisation & 27 & 29 \\
\hline Suprapubic catheterisation & 11 & 12 \\
\hline Unknown & 2 & 2 \\
\hline
\end{tabular}

One issue regarding the study design needs to be discussed. The reliability of the study would have improved if a questionnaire was answered by each individual gynaecologist rather than each hospital. However, we believe that the study reflects the routine clinical practice in The Netherlands accurately because of the high percentage of hospitals with a catheterisation protocol and the high response rate obtained in our study.

Most gynaecologists routinely administer prophylactic antibiotics before surgery. There is no evidence available to
Table 5 Indication for starting antibiotic treatment and diagnosis of urinary tract infections

Diagnostic criteria are given as minimal requirement to start antibiotic treatment

\begin{tabular}{lcc}
\hline Indication for starting antibiotic treatment $(n=92)$ & $n$ & Percent \\
\hline Total of group that starts antibiotic treatment without urinary test & 19 \\
Routinely during or after catheterisation & 15 \\
Symptoms without confirmation of urine analysis or culture & 4 \\
Total of group that starts antibiotic treatment relying on urine analysis (dipstick testing) & 61 & 66 \\
Urine analysis results & 33 \\
Urine analysis results or dipstick testing with complaints & 28 \\
Total of group that starts antibiotic treatment relying on urinary culture & 12 & 13 \\
Urinary culture results & 9 \\
Urinary culture with complaints & \multicolumn{2}{|c}{} \\
Diagnosis of urinary tract infection (dipstick) & 3 \\
Nitrite positive only & \multicolumn{2}{|c}{74} \\
Leucocyte positive only & 68 \\
Both nitrite and leucocytes positive & 0 & 0 \\
Diagnosis of urinary tract infection (urine culture) & 24 & 26 \\
$10^{3}$ colony-forming units & \multicolumn{2}{|c}{7} \\
$10^{4}$ colony-forming units & 14 & 15 \\
$10^{5}$ colony-forming units & 13 \\
\hline
\end{tabular}


support or reject such regimen with regard to infectious complications post-operatively [6]. With respect to the need of post-operative insertion of vaginal gauze for haemostatic reasons, there is no available evidence to support or reject routine insertion after the different types of vaginal surgery. There is limited evidence that $3 \mathrm{~h}$ of vaginal packing would be as sufficient for the prevention of post-operative haemorrhage or haematoma as vaginal packing for $24 \mathrm{~h}$ $[7,8]$.

The most frequently performed type of catheterisation was transurethral indwelling catheterisation. This was, on average, applied 3 days (range 1-7 days) in patients undergoing anterior repair. Based on two randomised trials, this standard duration of 3 days following anterior colporrhaphy appears to be too long as the majority of patients do not encounter voiding problems 1 day after surgery $[9,10]$. Only five hospitals applied an initial catheterisation period of 1 day post-operatively, implicating overtreatment in a significant proportion of patients.

After removal of the first catheter, the decision to recatheterise depends on the definition of abnormal PVR. Therefore, a question was included about the used definitions for PVR and the reliability of the methods to measure PVR.

Transurethral catheterisation will yield reliable representations of PVR only when the measurement is performed directly after voiding to minimise refilling due to continuing diuresis. Furthermore, there is minimal evidence that Foley catheters do not represent PVR as accurately as shorter catheters [11]. Studies evaluating the accuracy of bladder scanning devices for measuring PVR have reported good correlations with catheterised bladder volume [1214], although some conflicting data are available [15]. Together with the non-invasiveness of bladder scanning, it makes this procedure an appropriate method which is reflected by the high percentage of respondents using bladder scanning devices.

The wide range $(50-250 \mathrm{~mL})$ in cut-off points for considering PVR to be pathological reflects the absence of evidence or guidelines regarding this matter [16]. As abnormal PVR is a complication that necessitates additional treatment, the absence of consensus implies that part of the patients receive either undertreatment or overtreatment.

The choice for different types of catheterisation when abnormal PVR is diagnosed can depend on evidence, experience with a certain method and patient's preferences. The group that initially had chosen suprapubic catheterisation continued this treatment for obvious reasons. Suprapubic catheterisation is tolerated better than transurethral indwelling catheterisation and offers the possibility of clamping and voiding trials while the catheter stays in place [17]. Furthermore, the risk of urinary tract infection is lower when compared to transurethral indwelling catheterisation
[18]. However, the risk of complications while placing suprapubic catheters justifies a reserved attitude towards this technique [19]. This is especially the case after vaginal prolapse surgery after which the duration of initial catheterisation can be reduced to 1 day or even less [8-10]. Possibly, the small proportion of gynaecologists that initially inserts a suprapubic catheter reflects this notion.

A significant proportion of gynaecologists that initially applied transurethral indwelling catheterisation (77\%) did the same in case of retention (57\%); however, 17 of the 92 respondents changed to intermittent catheterisation $(18 \%)$.

This shift towards this treatment can possibly be explained by the advantage of intermittent catheterisation that treatment can be stopped immediately when retention is not present anymore and the finding that, in general, intermittent catheterisation exhibits a lower risk of bacteriuria compared to indwelling transurethral catheterisation [17]. Intermittent catheterisation, therefore, should be considered more often in the treatment of abnormal PVR. However, randomised studies are needed to determine the required duration of catheterisation and the consecutive risk of urinary tract infection with the two methods.

The last topic of our questionnaire addressed definitions and management of urinary tract infection. Based on the data from our study, it appears that patients undergoing catheterisation are exposed to a significant risk of unneeded treatment for urinary tract infection due to suboptimal implementation of evidence regarding the use of antibiotic treatment and diagnostic tests.

About one out of five responders routinely administer antibiotics during prolonged catheterisation or based on the presence of symptoms suggestive for urinary tract infection without additional diagnostic testing. Standard antibiotic treatment during or after removal of the catheter is not indicated in urogynaecology patients as many have no bacteriuria or appear to clear bacteriuria without antibiotics $[9,20]$. Treatment based on symptoms only seems also unjustified, as large prospective studies found similar incidences of symptoms in groups of patients with urinary tract infections and without urinary tract infections [2]. Although this study did not concern patients after prolapse surgery, it shows that the presence of the catheter itself can produce similar symptoms. Therefore, starting antibiotic treatment based on symptoms only is questionable.

Diagnostic tests applied to screen for the presence of urinary tract infection in Dutch laboratories mostly comprise urine dipstick testing. When indicated, sedimentation is performed or the urine is cultured. The combined observation of both a negative nitrite and negative leucocyte esterase test result when performing dipstick testing has the highest predictive value to exclude the presence of bacteriuria $[21,22]$. That our study shows that $74 \%$ of the responders define the presence of urinary tract 
infection in case of the presence of nitrite only demonstrates that possibly many patients are treated with antibiotics without proper diagnosis.

In conclusion, practice variation of catheterisation regimes following POP surgery probably exists due to limited evidence on this subject and to suboptimal implementation of the evidence that is available. Consequences of this practice variation are unnecessary prolonging of catheterisation, an increase of urinary tract infections related to prolonged catheterisation, overadministration of antibiotics, prolonged hospitalisation and, consequently, increased costs. Well-designed studies are urgently needed to optimise catheterisation management and to develop evidence-based definitions of abnormal PVR and urinary tract infections.

\section{Conflicts of interest None.}

Open Access This article is distributed under the terms of the Creative Commons Attribution Noncommercial License which permits any noncommercial use, distribution, and reproduction in any medium, provided the original author(s) and source are credited.

\section{References}

1. Saint S, Wiese J, Amory JK, Bernstein ML, Patel UD, Zemencuk JK et al (2000) Are physicians aware of which of their patients have indwelling urinary catheters? Am J Med 109:476-480

2. Tambyah PA, Makis DG (2000) Catheter-associated urinary tract infection is rarely symptomatic: a prospective study of 1,497 catheterized patients. Arch Intern Med 160:678-682

3. Jain P, Parada JP, David A, Smith LG (1995) Overuse of the indwelling urinary tract catheter in hospitalized medical patients. Ann Intern Med 155:1425-1429

4. Pratt RJ, Pellowe C, Loveday HP, Robinson N, Smith GW, Barrett $S$ et al (2001) Department of Health (England).The epic project: developing national evidence-based guidelines for preventing healthcare associated infections. Phase I: Guidelines for preventing hospital-acquired infections. Department of Health (England). J Hosp Infect 47 Suppl:S3-82

5. Hilton P (1988) Bladder drainage: a survey of practices among gynaecologists in the British Isles. BJOG 95:1178-1189

6. Phipps S, Lim YN, McClinton S, Barry C, Rane A, N'Dow J (2006) Short term urinary catheter policies following urogenital surgery in adults. Cochrane Database Syst Rev 2:CD004374 Review

7. Ottesen M, Sørensen M, Rasmussen Y, Smidt-Jensen S, Kehlet H, Ottesen B (2002) Fast track vaginal surgery. Acta Obstet Gynecol Scand 81:138-146
8. Glavind K, Morup L, Madsen H, Glavind J (2007) A prospective, randomised, controlled trial comparing 3 hour and 24 hour postoperative removal of bladder catheter and vaginal pack following vaginal prolapse surgery. Acta Obstet Gynecol Scand $86: 1122-1125$

9. Schiøtz HA, Tanbo TG (2006) Postoperative voiding, bacteriuria and urinary tract infection with Foley catheterization after gynaecological surgery. Acta Obstet Gynecol Scand 85:476-481

10. Hakvoort RA, Elberink R, Vollebregt A, Ploeg T, Emanuel MH (2004) How long should urinary bladder catheterisation be continued after vaginal prolapse surgery? A randomised controlled trial comparing short term versus long term catheterisation after vaginal prolapse surgery. BJOG 111:828-830

11. Haylen BT, Frazer MI, MacDonald JH (1989) Assessing the effectiveness of different urinary catheters in emptying the bladder: an application of transvaginal ultrasound. $\mathrm{Br} \mathrm{J}$ Urol 64:353-356

12. Fuse H, Yokoyama T, Muraishi Y, Katayama T (1996) Measurement of residual urine volume using a portable ultrasound instrument. Int Urol Nephrol 28:633-637

13. Bent AE, Nahhas DE, McLennan MT (1989) Portable ultrasound determination of urinary residual volume. Am J Obstet Gynecol 160:1013-1016

14. Goode PS, Locher JL, Bryant RL, Roth DL, Burgio KL (2000) Measurement of postvoid residual urine with portable transabdominal bladder ultrasound scanner and urethral catheterization. Int Urogynecol J Pelvic Floor Dysfunct 11:296-300

15. Fedorkow DM, Dore S, Cotton A (2005) The use of an ultrasound bladder scanning device in women undergoing urogynaecologic surgery. J Obstet Gynaecol Can 27:945-948

16. Abrams P, Cardozo L, Fall M, Griffiths D, Rosier P, Ulmsten U, Standardisation Sub-Committee of the International Continence Society et al (2003) The standardisation of terminology in lower urinary tract function: report from the standardisation subcommittee of the International Continence Society. Urology 61:37-49

17. Niël-Weise BS, van den Broek PJ (2005) Urinary catheter policies for short-term bladder drainage in adults. Cochrane Database Syst Rev 3:CD004203 Review

18. Andersen JT, Heisterberg L, Hebjørn S, Petersen K, Stampe Sørensen S, Fischer-Rasmussen W, Mølsted Pedersen L, Nielsen NC (1985) Suprapubic versus transurethral bladder drainage after colposuspension/vaginal repair. Acta Obstet Gynecol Scand 64:139-143

19. Ahluwalia RS, Johal N, Kouriefs C, Kooiman G, Montgomery BS, Plail RO (2006) The surgical risk of suprapubic catheter insertion and long-term sequelae. Ann R Coll Surg Engl 88:210-213

20. Summitt RL Jr, Stovall TG, Bran DF (1994) Prospective comparison of indwelling bladder catheter drainage versus no catheter after vaginal hysterectomy. Am J Obstet Gynecol 170:1815-1818 discussion 1818-21

21. Devillé WL, Yzermans JC, van Duijn NP, Bezemer PD, van der Windt DA, Bouter LM (2004) The urine dipstick test useful to rule out infections. A meta-analysis of the accuracy. BMC Urol 4:4

22. St John A, Boyd JC, Lowes AJ, Price CP (2006) The use of urinary dipstick tests to exclude urinary tract infection: a systematic review of the literature. Am J Clin Pathol 126:428-436 